\title{
Cued Zone Stimuli Simulation and Model Analysis
}

\author{
Ms. Elena Rosemaro \\ Department of Management Studies, \\ VIM Australia
}

Article Info

Page Number: 105 - 107

Publication Issue:

Vol 71 No. 1 (2022)

\section{Article History}

Article Received: 18 November 2021

Revised: 03 December 2021

Accepted: 19 December 2021

Publication: 28 January 2022

\begin{abstract}
Attention may be a significant precursor to visual awareness but does not fulfil the sufficiency test. Moreover, the study uses a Posner endogenous spatial cueing paradigm to show that the time taken by the subject to discriminate the point of reference of a stimulus is minimized if the individual is signal towards the location of the stimulus. The reaction-time advantage is achieved without any reduction in discrimination accuracy. It implies that it cannot be said to have been caused by the speed-error trade off or distinctions in bias between the cued and uncued locations. Therefore, the subject was unaware of the stimuli to which processing was attenionally directed. In the end, the researchers conclude that attention is not a satisfactory condition for awareness.
\end{abstract}

Keywords: - Simulation, Reaction-Time etc.

\section{Introduction}

This research is a follow-up study on the researcher's previous study that involved a patient that had a neurological condition of blindsight in order to investigate whether attention may not be an enough condition for awareness. Notably, this research now considers the question about the sufficiency condition. Under a modified model, the scholars now sought to use discrimination instead of detection as the subject's task. Within the discrimination task, trials on which targets are not present are unnecessary. Therefore, the cue-validity is only important on each trial and the impact of any bias directed towards a response can be managed through counterbalancing. Therefore, the present research sought to determine whether the pros of reaction-time (RT) in the previous research could have been attributed to speed-error trade-off or caused by spatially selective attention.

Apart from the significant change in task from detection to discrimination, the present study varies the time taken in the presentation of cues and targets. The researchers note that in an endogenous cueing task as the one that they have evaluated, the efficiency of the cues in the typical subjects rises as the time taken before the onset of the cue and the onset of the target widens. Thus, the study set random intervals of between 200 and $450 \mathrm{~ms}$ to evaluate whether the same variation in cue effectiveness could be established in the subject GY. Critically, the researchers also never sought that GY makes trial-by-trial comments on the target awareness within this study. The purpose of this was to make the response requirements simpler just in case making a commentary decision affected his reaction to the orientation of the target. Rather, the research sought to use oral reports of awareness in pre-tests. 


\section{Methodology}

The strength of the use of the Posner Cueing Task methodology is that it carries out a neuropsychological test that can be used to evaluate attention. It significantly helped in examining a person's ability to carry out attentional shift as well as assessing its role in determining spatial attention. Therefore, the authors carefully used this method to elicit, measure and theoretically characterize the attentional orientation of a person. While this is the case, it is also pointed out that the Posner cuing paradigm method may not guarantee an unambiguous evaluation of attention (Kentridge, Heywood and Weiskrantz, 2004). Therefore, the method used engages a subject named GY who is 41 years old and has in the past suffered unilateral damage to the left striate cortex that has been established by computerised tomography and magnetic resonance imaging (MRI). This could be attributed to a motor vehicle accident suffered at the age of eight years old. The subject also has a right homonymous hemianopia and has approximately $3^{\circ}$ of macular sparing that can easily be explained from the imaging of the neuro regions. Importantly, the subject matter agreed to participate in the study through informed consent. Essentially, the significance of using the subject matter is that it guarantees continuity as this individual had been used in a previous study pursuing the same objective as this one. Additionally, the use of GY coupled with an improved apparatus and procedure would lead in removing the ambiguities that was registered in the previous study by the authors.

Stimuli was created using a Cambridge Research Systems VSG2/3 and showed against the backdrop of 51.4 cd m-2 background, using a Samtron SC-726 GXL colour monitor (Kentridge, Heywood and Weiskrantz, 2004). The system's response box was also utilized to collate the responses as well as generate the auditory signals to the subject. All the trials commenced with a $150 \mathrm{~ms}$ presentation of a black arrow $0.77^{\circ}$ in length that is directed at one of the two probable locations (Kentridge, Heywood and Weiskrantz, 2004). At the end of this interval, target presentation was started. Notably, before the commencement of any of these events, every trial was explained to the subject GY and was allowed to see some of the trials within the visual field that had not been impaired. Further, the subject had to respond promptly on the response box as soon as he heard the sound of the tone to show whether the target was horizontal or vertical.

\section{Results and Discussion}

The results of this study revealed that there is evidence that clearly shows that performance advantage can lead to the processing of stimuli that is offered at a cued place without the stimuli producing awareness. Further, it is imperative that attention hastens the processing of locations as much as they cannot be seen. These results indicate that the subject can take advantage of a temporal alerting cue to target. Moreover, the subject can direct his attention to an unseen location. Significantly, the investigation revealed that GY's lesion may have affected the vision, but it is improbable that it would affect the parietal or other neural representation of space that play a critical role in determining spatial selective attention. In reality, this study confirmed that visual spatial attention is not a full condition for visual awareness.

The authors have succeeded in addressing the paradox of blindsight patients being able to react to "unseeable" location by using this 41-year-old subject under an improved experimental framework. First, they have reaffirmed that blindsight is a condition whereby patients that have damage to their primary visual contexts still maintain the ability to detect. They can also discriminate and localize the visual stimuli that are present in the location of their visual fields. This occurs irrespective of the fact that they may argue that they are subjectively blind. While a past research study or investigation had showed that the blindsight patient GY had a reaction-time (RT) advantage for the detection of targets, the classic Posner method may be 
inadequate in this case (Kentridge, Heywood and Weiskrantz, 2004). In so doing, they also established that the performance advantage did not also come together with awareness of targets in the cued location.

\section{Conclusion}

Therefore, it sought to show that attention to target is not a fully sufficient condition that one is aware of the target. In the end, it can be argued that this study is more comprehensive than the previous ones over the same issue as it better explains blindsightedness in a patient.

\section{Reference}

[1]. Kentridge, R. W., Heywood, C. A., \& Weiskrantz, L. (2004). Spatial attention speeds discrimination without awareness in blindsight. Neuropsychologia, 42(6), 831-835.

[2].Harsh, S. ., Singh , D., \& Pathak , S. (2022). Efficient and Cost-effective Drone - NDVI system for Precision Farming. International Journal of New Practices in Management and Engineering, 10(04), 14-19. https://doi.org/10.17762/ijnpme.v10i04.126

[3].Krishnan, S. ., and D. B. . Prasanthi. "Prediction of CPU Utilization in Cloud Environment During Seasonal Trend". International Journal on Recent and Innovation Trends in Computing and Communication, vol. 9, no. 12, Dec. 2021, pp. 08-11, doi:10.17762/ijritcc.v9i12.5493.

[4].Faisal, M. R. "Software Testing: Data Kart and Integrated Pipeline Approach: Data Kart and Integrated Pipeline Approach for Software Testing”. International Journal on Recent and Innovation Trends in Computing and Communication, vol. 9, no. 8, Sept. 2021, pp. 01-04, doi:10.17762/ijritcc.v9i8.5480. 